\title{
The effect of fiber oxidation on the friction and wear behaviors of short-cut carbon fiber/polyimide composites
}

\author{
X. R. Zhang ${ }^{1,2}, X . Q . P e i^{1,2}, Q . H$. Wang $^{1 *}$ \\ ${ }^{1}$ State Key Laboratory of Solid Lubrication, Lanzhou Institute of Chemical Physics, Chinese Academy of Sciences, \\ Lanzhou 730000, China \\ ${ }^{2}$ Graduate School, Chinese Academy of Sciences, Beijing 100039, China
}

Received 16 March 2007; accepted in revised form 29 April 2007

\begin{abstract}
Pitch-based short-cut carbon fibers were treated by $\mathrm{HNO}_{3}$ oxidation, thereafter the treated (CFN) and untreated carbon fibers (CF) were incorporated into polyimide (PI) matrix to form composites. The carbon fibers before and after treatment were examined by Fourier Transform Infrared Spectroscopy (FTIR), X-ray photoelectron spectroscopy (XPS) and scanning electron microscope (SEM). The friction and wear behaviors of PI composites sliding against GCr15 steel rings were evaluated on an M-2000 model ring-on-block test rig, which revealed that small incorporation of carbon fibers can decrease the friction coefficient and improve the wear resistance of PI composites, and that the reinforcement effect of treated carbon fibers was better than that of the untreated ones. It was found that the optimum content of carbon fibers is $15 \mathrm{wt} \%$ when a thin and continuous transfer film was formed on the counterpart surface during the friction process. With further increasing content of carbon fibers, the friction coefficient increased and the wear resistance reduced owing to the drop out of carbon fibers from PI matrix. Besides, the friction coefficient of the PI composites decreased and the wear resistance improved with increasing load, while for the pure PI, its wear resistance decreased drastically owing to the micro-melting and mechanical deterioration caused by friction heat under a higher load.
\end{abstract}

Keywords: polymer composites, reinforcement, carbon fiber, surface treatment, friction and wear

\section{Introduction}

Carbon fiber reinforced polymer composites have been widely used in the fields of aerospace and high technical product. Carbon fibers present extremely high strength and modulus, good stiffness, and creep resistance etc., and have been widely employed as the reinforcing materials in the high performance polymer composites which have been extensively used in many industrial fields [1]. However, when applied without previous surface modification, the physical-chemical interaction between carbon fibers and its reinforced matrix is not tough enough due to the inert surface property of carbon fibers, which will directly affect the degree of interfacial adhesion in the composite sys- tem. Numerous methods concerning surface treatment, such as chemical method [2-4], electrochemical method [5-7], plasma treatment [8,9], etc., have been developed to increase the quantity of surface functional groups and thus enhance the ability to establish strong interactions between fibers and matrix.

Polyimide (PI) possesses some extraordinary characteristics, such as excellent mechanical and electrical (insulating) properties, good thermal stability and chemical inertness, high wear resistance, and resistance against high energy radiation $[10,11]$. The fiber-reinforced polyimide composites with high performance have especial applications in aerospace, robots, sports goods, etc. By reinforcing 
PI with carbon fibers of high strength and modulus, excellent heat stability, and chemical inertness [12], it might be feasible to develop high performance PI-based composites. In the past years, many researchers have focused on the tribological behaviors of carbon fibers filled composites in various directions. Lu and Friedrich [13, 14] studied systematically the influence of carbon fibers volume content on the friction and wear behaviors of their composites, and declared that an optimum range for short carbon fibers in PEEK matrix is $15-25 \%$ (volume) according to the improved specific wear rate.

The objective of this work is to study the friction and wear behaviors of the modified short-cut carbon fibers reinforced polyimide composites. The surface properties of the carbon fibers before and after treatment were compared by FTIR, SEM, XPS. This work is believed to be helpful for the understanding of the effect of $\mathrm{HNO}_{3}$ oxidation.

\section{Experimental}

\subsection{Materials}

PI(YS-20) powders $(<38 \mu \mathrm{m})$ were commercially obtained from Shanghai Synthetic Resin Institute (Shanghai, China). The short-cut carbon fiber was about $100 \mu \mathrm{m}$ in length and $7.8 \mu \mathrm{m}$ in diameter, and the density was about $1.8 \mathrm{~g} / \mathrm{cm}^{3}$. $\mathrm{HNO}_{3}$ solution (AR, with the weight concentration of 65$68 \%)$.

\subsection{Preparation of PI composites}

The commercial short-cut carbon fibers were dipped in acetone for $24 \mathrm{~h}$, then cleaned ultrasonically with acetone for $0.5 \mathrm{~h}$. Finally, they were dried at $100^{\circ} \mathrm{C}$ for $5 \mathrm{~h}$ before used. The oxidation of carbon fibers was achieved by heating carbon fibers in nitric acid (with the weight concentration of $65-68 \%$ ) at $90^{\circ} \mathrm{C}$ for $1.5 \mathrm{~h}$. After the surface oxidation, the modified fibers were thoroughly washed with distilled water, and dried before used. Such modified fibers were named as $\mathrm{CFN}$.

In order to investigate the possible change of chemical composition of the carbon fibers treated by $\mathrm{HNO}_{3}$ oxidation, Fourier Transform Infrared Spectroscopy (FTIR) measurements in the mid infrared (4000-400 $\mathrm{cm}^{-1}$ ) were performed, which were recorded on a Bruker IFS/66v spectrometer. The specimens for FTIR measurements were prepared by $\mathrm{KBr}$ tabletting method. X-ray photoelectron spectroscopy (XPS) was used to detect functionalities on activated carbon fibers surfaces. The XPS measurement was performed using VG Scientific ESCA LAB 210 spectrometer, the base pressure in the sample chamber was about $10^{-7} \mathrm{~Pa}$. The morphologies of the untreated carbon fibers (CF) and $\mathrm{HNO}_{3}$ treated carbon fibers (CFN) were compared by SEM observation, before which the tested carbon fibers specimens were plated with gold coating to render them electrically conductive.

The CF and CFN were mixed with PI powders at selected mass fractions to prepare mixtures for the preparation of PI composites. To prepare the PI composites blocks, the mixtures were compressed and heated to $380^{\circ} \mathrm{C}$ in a mould. The pressure was held at $40 \mathrm{MPa}$ for $75 \mathrm{~min}$ to allow full compression sintering. At the end of each run of compression sintering, the resulting specimens were cooled with the stove in air, cut into pre-set sizes for friction and wear tests.

\subsection{Friction and wear test}

The friction and wear behaviors of PI composites sliding against stainless steel were evaluated on an M-2000 model ring-on-block test rig (made by Jinan Testing Machine Factory, China).The contact schematic diagram is shown in Figure 1, the blocks in a size of $30 \mathrm{~mm} \times 7 \mathrm{~mm} \times 6 \mathrm{~mm}$ were made of the PI composites, the rings of $\varnothing 40 \mathrm{~mm} \times 16 \mathrm{~mm}$ were made of GCr15 stainless steel, the chemical composition of the GCr15 bearing steel (mass fraction, $\%$ ) is shown in Table 1 . The tests were carried out

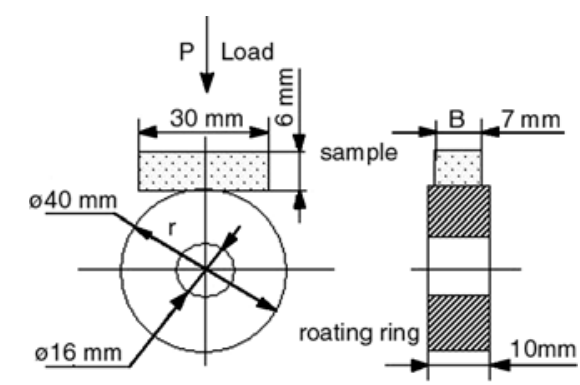

Figure1. The contact schematic diagram for the friction couple

Table 1. Chemical composition of the GCr15 steel ring

\begin{tabular}{|c|c|c|c|c|c|}
\hline \multicolumn{6}{|c|}{ Chemical composition (mass fraction, \%) } \\
\hline C & Mn & Si & P & S & Cr \\
\hline $0.95-1.05$ & $0.25-0.45$ & $0.15-0.35$ & $\leq 0.025$ & $\leq 0.025$ & $1.40-1.65$ \\
\hline
\end{tabular}


at a linear velocity of $0.431 \mathrm{~m} / \mathrm{s}$ in a period of 120 min with the loads ranging from $200 \mathrm{~N}$ to $500 \mathrm{~N}$. Before each test, the stainless steel ring and the PI composite block were polished to a roughness $\left(R_{a}\right)$ of about $0.2-0.3 \mu \mathrm{m}$. The block specimen was static and the GCr15 bearing was sliding against the block unidirectionally. The friction force was measured using a torque shaft equipped with strain gauges mounted on a vertical arm that carried the block, which was used to calculate the friction coefficient by taking into account the normal load applied. The width of the wear tracks was measured with a reading microscope to an accuracy of $0.01 \mathrm{~mm}$. Then the specific wear rate $(\omega)$ of the specimen was calculated from Equation (1).

$\omega=\frac{B}{L \cdot P}\left[\frac{\pi r^{2}}{180} \arcsin \left(\frac{b}{2 r}\right)-\frac{b}{2 r} \sqrt{r^{2}-\frac{b^{2}}{2}}\right]\left[\frac{\mathrm{mm}^{3}}{\mathrm{~N} \cdot \mathrm{m}}\right]$

where $B$ is the width of the specimen [mm], $r$ is the semi diameter of the stainless steel ring $[\mathrm{mm}]$, and $b$ is the width of the wear trace [mm], $L$ is the sliding distance in meter, $P$ is the load in Newton. The tests were repeated three times, the wear tracks of the composite and stainless steel specimens were examined on a JSM-5600LV scanning electron microscope (SEM). In order to increase the resolution for the SEM observation, the tested composite specimens were plated with gold coating to render them electrically conductive.

\section{Results and discussion}

\subsection{FTIR analysis of CF and CFN}

It can be seen from Figure 2 that there were many active groups on the surface of CFN. The peak in the $1100-1220 \mathrm{~cm}^{-1}$ region is associated with ether type structures $(\mathrm{C}-\mathrm{O}-\mathrm{C})$. The peak in the 1350 $1390 \mathrm{~cm}^{-1}$ region reveals the existence of $\mathrm{C}-\mathrm{N}$ bands. The peak in the $1500-1680 \mathrm{~cm}^{-1}$ region is assigned to aromatic $\mathrm{C}=\mathrm{C}$ bands and various substitution modes of the aromatic ring. The peak in the $1710 \mathrm{~cm}^{-1}$ region reveals evidence of carbonyl groups on the CFN surface. So it can be concluded that the structure of carbon fiber had changed and more polar components were formed.

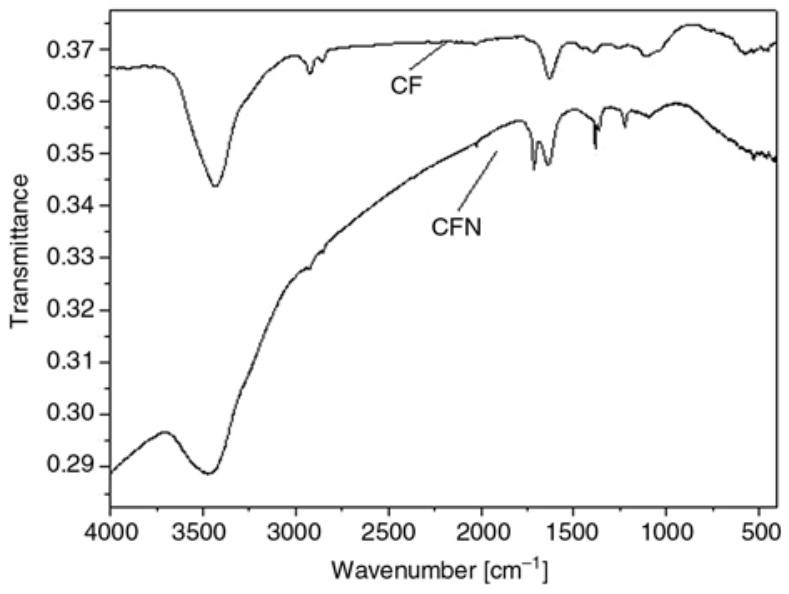

Figure 2. FTIR spectra of CF and CFN

\subsection{XPS analysis of CF and CFN}

The C1s XPS spectra of CF and CFN are shown in Figure 3. The dominant peak at $284.8 \mathrm{eV}$ was assigned to graphitic carbon in the fiber [15]. The peak at $286.2 \mathrm{eV}$ was assigned to the carbon in -C-O- structures. The binding energy of carbon in carbonyl groups is $287.5-288.1 \mathrm{eV}$.

It can be found from Table 2 that the surface $\mathrm{C}$ element concentration of the untreated, nitric acid treated carbon fiber samples were $80.2 \%, 67.4 \%$ respectively. A lower $\mathrm{C}$ concentration was found on the surfaces of the treated carbon fiber samples compared with that of the untreated ones. The surface $\mathrm{O}$ concentration of the untreated, nitric acid treated carbon fiber samples were $17.7 \%, 28.3 \%$, respectively. The $\mathrm{O} / \mathrm{C}$ ratio on the surfaces of the treated carbon fiber samples increased compared with the untreated ones.

After $\mathrm{HNO}_{3}$ oxidation, there were more active groups $(-\mathrm{O}-\mathrm{C}--\mathrm{C}=\mathrm{O}-\mathrm{O}-\mathrm{C}=\mathrm{O})$ on the surfaces of the carbon fibers, which increased total surface energy and their polarity and thus enhanced the

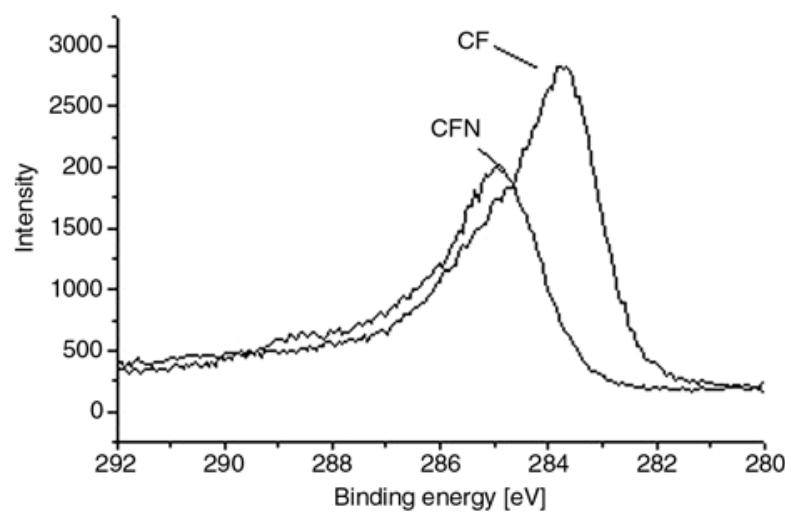

Figure 3. XPS spectra for $\mathrm{C} 1 \mathrm{~s}$ states on the $\mathrm{CF}$ and $\mathrm{CFN}$ 
Table 2. XPS surface element analysis data of CF and CFN

\begin{tabular}{|l|c|c|c|c|}
\hline Carbon fibers & $\mathbf{C}[\%]$ & $\mathbf{N}[\%]$ & $\mathbf{O}[\%]$ & $\mathbf{O} / \mathbf{C}$ \\
\hline Untreated & 80.2 & 2.1 & 17.7 & 0.22 \\
\hline Nitric acid treated & 67.4 & 4.3 & 28.3 & 0.42 \\
\hline
\end{tabular}

wettability of the carbon fibers with the PI matrix. Better wetting can improve the adhesive bond strength by increasing the thermodynamic work of adhesion or by reducing the number of interfacial defects [16]. Adhesion between the carbon fibers and PI matrix improved, which played an important role on the tribological properties of carbon fibers reinforced polymer composites.

\subsection{Morphology analysis of CF and CFN}

The morphologies of CF and CFN are shown in Figure 4. It can be seen that the surface of the carbon fiber after $\mathrm{HNO}_{3}$ oxidation treatment (Figure 4b) was rougher than that of the untreated one (Figure 4a). There appeared more defects, closely spaced grooves, protuberances and dongas on the surfaces of CFN, which can increase surface roughness of carbon fibers. This would provide more contacts between the carbon fibers and PI matrix to ensure a significant level of van der Waals force, which enhanced the degree of mechanical interlocking between the fibers and the PI matrix [17].
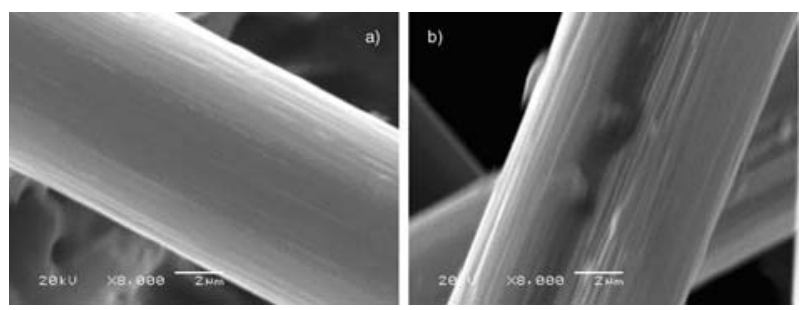

Figure 4. SEM morphologies of the CF (a) and CFN (b)

\subsection{The friction and wear properties}

\subsubsection{The influence of content of carbon fibers on the friction and wear properties of the PI composites}

Figure 5 shows the friction coefficient and specific wear rate of four PI composites at a sliding speed of $0.431 \mathrm{~m} / \mathrm{s}$ under $200 \mathrm{~N}$. It was found that the small incorporation of carbon fibers can decrease the friction coefficient and improve the wear resistance of PI composites. Meanwhile, the results revealed that the reinforcement effect of the treated carbon fibers
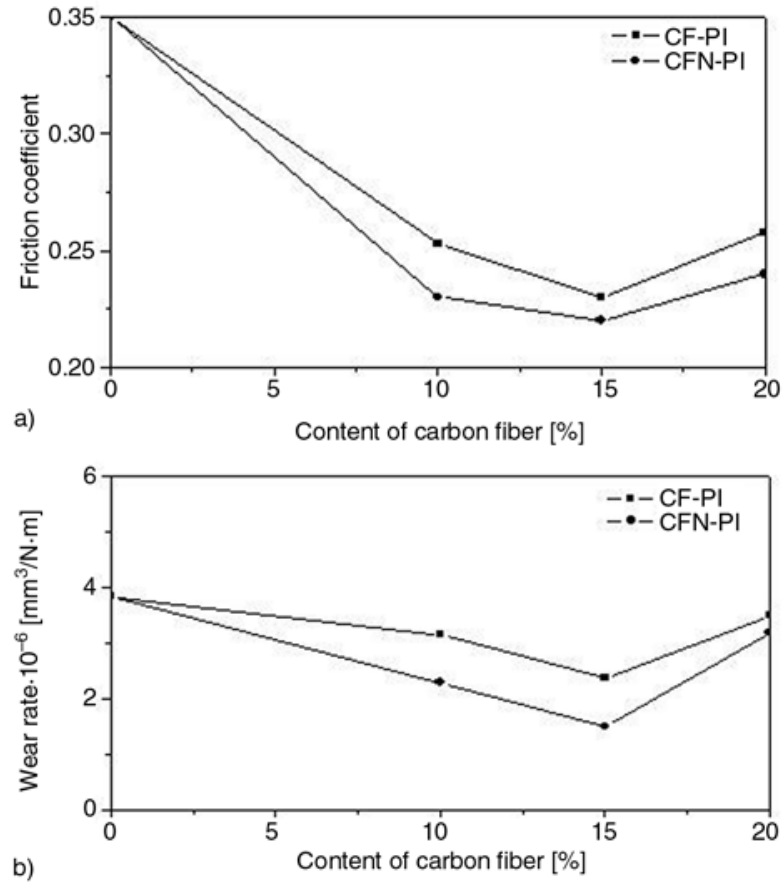

Figure 5. Variation of the friction coefficient and wear rate of the PI composites. a) friction coefficient vs. mass content of carbon fiber, b) wear rate $v s$. mass content of carbon fiber

was better than that of the untreated ones. The optimum content of carbon fibers is $15 \mathrm{wt} \%$ when a thin and continuous transfer film was formed on the counterpart surface during the friction process. It was also found that the friction coefficient of the CFN-PI composites decreased slightly than that of the CF-PI composites, though the wear resistance improved drastically.

With increase in content, it also can be found that the friction coefficient increased slightly and the wear resistance reduced. With a high content of carbon fibers, there appeared more PI debris and broken carbon fibers fragments on the worn surface (this was supported by SEM as discussed in the subsequent section) owing to the drop out of carbon fibers from PI matrix during the friction process, which revealed that the adhesion between the carbon fibers and PI matrix decreased and there appeared more defects. This shifted the wear mechanisms from adhesive wear to abrasive wear, which reduced the wear resistance drastically and made the friction coefficient to have an increasing trend. However, the high fraction of carbon fibers made the composites stiffer, the ability of resistance against cracking improved, which made the friction coefficient decrease. So the friction coefficient of the composites increased slightly. 


\subsubsection{The influence of load on the friction and wear properties of the CFN-PI composites}

Figure 6 shows the influence of load on the friction coefficient and specific wear rate of the CFN-PI composites. It can be seen that the friction coefficient of the pure PI decreased slightly with increasing load when the load was below $300 \mathrm{~N}$. At the same time, the specific wear rates of the pure PI increased slowly. When the load beyond this range, the friction coefficient decreased drastically and the specific wear rate increased greatly owing to the micro-melting and mechanical deterioration caused by friction heat under a higher load. High temperature during friction process can cause an increase in the real contact areas and a decrease in mechanical strength of the PI composites, now, the latter takes a dominant place. As for the modified carbon fibers reinforced PI composites, both the friction coefficient and the specific wear rates of the CFN-PI composites decreased because the transfer film can form easier owing to a gradual increase of the real contact areas and the increase in adhesive force between the film and counterpart.
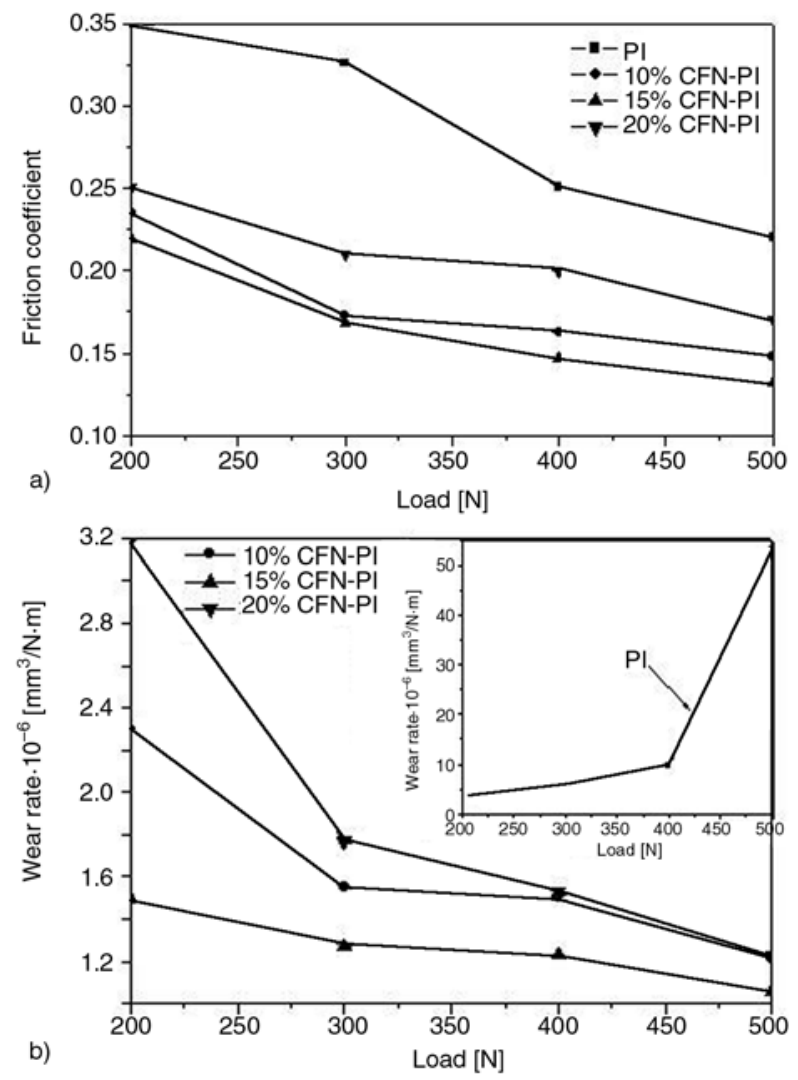

Figure 6. Variation of the friction coefficient and the wear rate of the CFN-PI composites under different load. a) Friction coefficient vs. load, b) wear rate vs. load

\subsubsection{The influence of sliding time on the friction and wear properties of the CFN-PI composites}

It can be seen from Figure 7 that the frictional process is composed of two distinct stages: the one is the running-in period and the other is the steadystate period. The friction coefficient of the runningin period was obviously higher than that of the steady-state period. It took less than $15 \mathrm{~min}$ to transit from the running-in period to the steady-state period. Because of the ability of self-lubricating of carbon fibers, the PI matrix transferred to the counterpart surface easier and the transfer film can form easier. After the formation and the peeling-off of the transfer film came to a balance, the friction coefficient became stable [10]. Moreover, it can be found that the time of running-in period of pure PI was obviously longer than that of the carbon fibers reinforced PI composites and the friction curve was more fluctuant, which corresponded to its poorer wear resistance under a higher load.

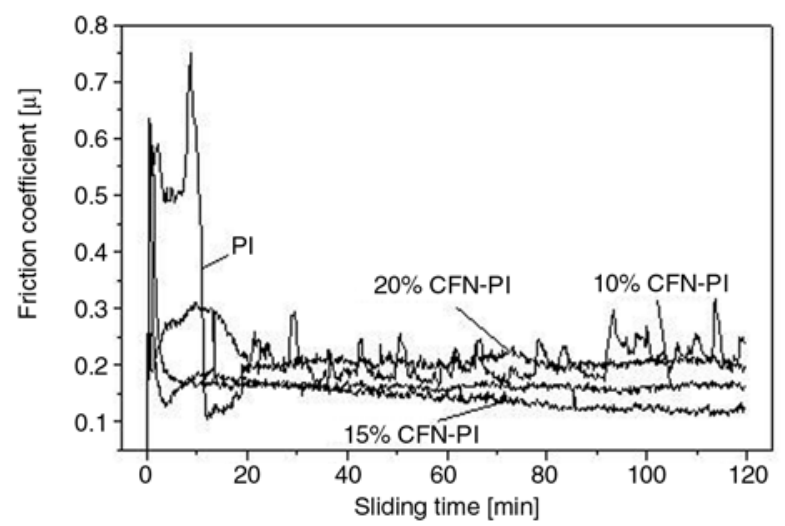

Figure 7. Typical friction coefficient variation of the CFN-PI composites with the sliding time $(0.431 \mathrm{~m} / \mathrm{s}$ and $400 \mathrm{~N})$

\subsection{SEM analysis of the worn surfaces and the transfer films on the surface of GCr15 steel rings}

Figure 8 shows the SEM morphologies of the worn surfaces of the PI composites sliding against the GCr15 steel at a sliding speed of $0.431 \mathrm{~m} / \mathrm{s}$ under $200 \mathrm{~N}$. The worn surface of the pure PI was characterized by severe plastic deformation and microcracking (Figure 8a), while a large amount of transferred PI debris were observed on the stainless steel counterpart surface and the transfer film was thick and discontinuous (Figure 9a), which correspond to its high friction coefficient of the pure PI. 

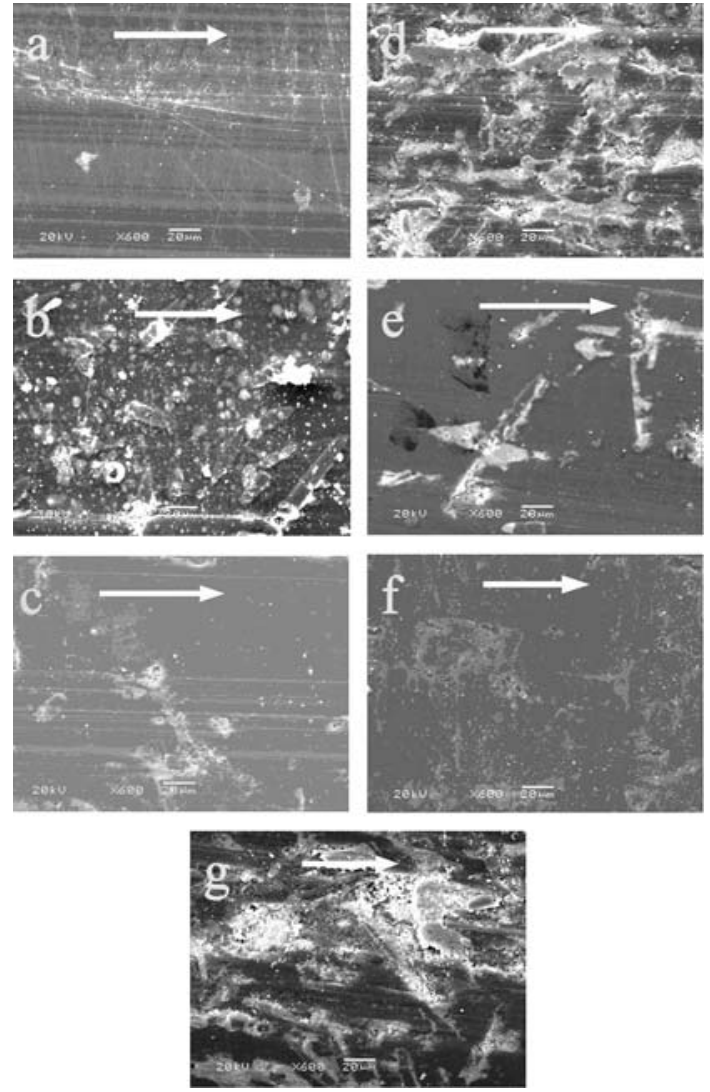

Figure 8. SEM morphologies of the worn surface of PI composites (600x); a) pure PI, b) $10 \%$ CF-PI, c) $15 \%$ CF-PI, d) $20 \%$ CF-PI, e) $10 \%$ CFN-PI, f) $15 \% \mathrm{CFN}-\mathrm{PI}, \mathrm{g}) 20 \% \mathrm{CFN}-\mathrm{PI}(L=200 \mathrm{~N}$, $V=0.431 \mathrm{~m} / \mathrm{s}$ ) (arrow indicates the sliding direction)

In contrast, the $10 \mathrm{wt} \% \mathrm{CF}-\mathrm{PI}$ composite was characterized by slight scuffing and spalling and there was no obvious plastic deformation (Figure 8b) and the transfer film was thick and discontinuous (Figure $9 b$ ). The scuffing and spalling phenomena on the worn surface was significantly abated when the content of carbon fibers was $15 \mathrm{wt} \%$ (Figure 8c), meanwhile, the transfer film became thinner and uniform (Figure 9c). However, the worn surface of the PI composite with 20 wt\% carbon fibers (Figure $8 \mathrm{~d}$ ) was characterized by many pulling-out and exposures of the carbon fibers and there were more wear debris and carbon fiber fragments, which indicated the poor adhesion between the carbon fibers and PI matrix. The drop out of the carbon fibers from PI matrix shifted the wear mechanisms from adhesive wear to abrasive wear. Meanwhile, the transfer film (Figure 9d) appeared to be thick, rough and discontinuous on the GCr15 steel counterpart surface.
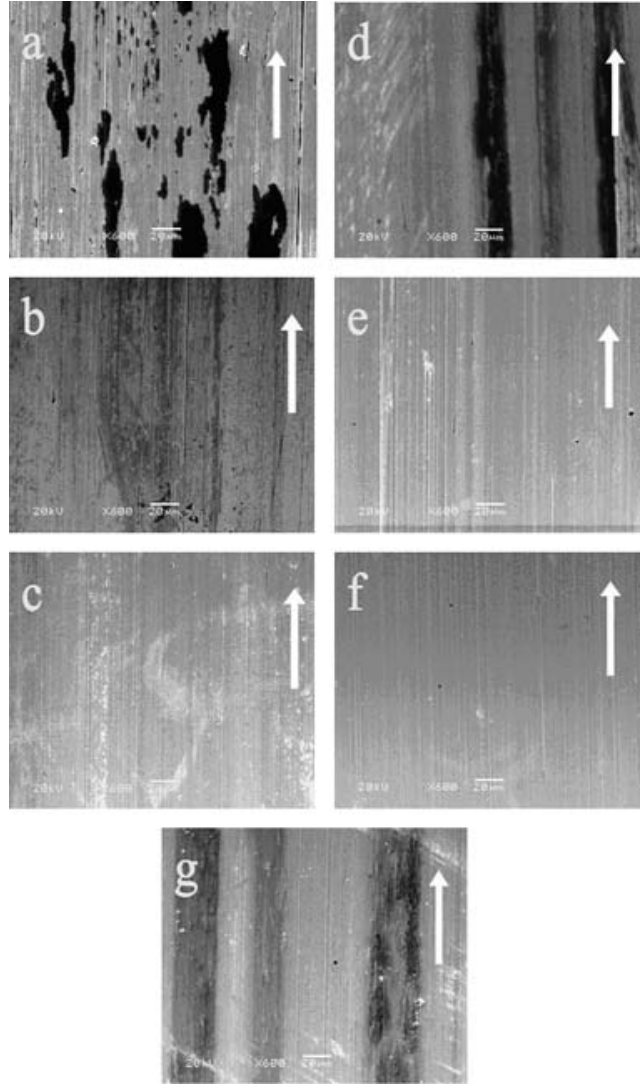

Figure 9. SEM morphologies of the transfer films on the surface of GCr15 steel counterpart (600x). a) pure PI, b) $10 \%$ CF-PI, c) $15 \%$ CF-PI, d) $20 \%$ CF-PI, e) $10 \%$ CFN-PI, f) $15 \%$ CFN-PI, g) $20 \%$ CFN-PI (arrow indicates the sliding direction)

It can be seen that the worn surface of the $10 \mathrm{wt} \%$ CFN-PI composites (Figure 8e) was characterized by a few cracks and fibers fragments and the transfer film (Figure 9e) was thick and continuous. There was no PI debris and fibers fragments on the worn surface of the $15 \mathrm{wt} \%$ CFN-PI composites (Figure 8f) and the transfer film (Figure 9f) is thinnest and continuous. It also can be seen that there were many exposures and wear debris on the worn surface of $20 \mathrm{wt} \%$ CFN-PI composites (Figure $8 \mathrm{~g}$ ) and the transfer film (Figure 9g) was thicker and discontinuous on the GCr15 steel counterpart surface. It can be concluded that the worn surfaces of the CFN-PI composites and the counterparts surfaces were smoother than the CF-PI composites with the same carbon fibers content and the PI matrix combined the carbon fiber better, the transfer film of $15 \mathrm{wt} \%$ CFN-PI composites (Figure 9f) appeared to be thinner, smoother and much more continuous, which conformed to the smaller friction coefficient and better wear resistance of the CFN-PI composites than CF-PI composites. 

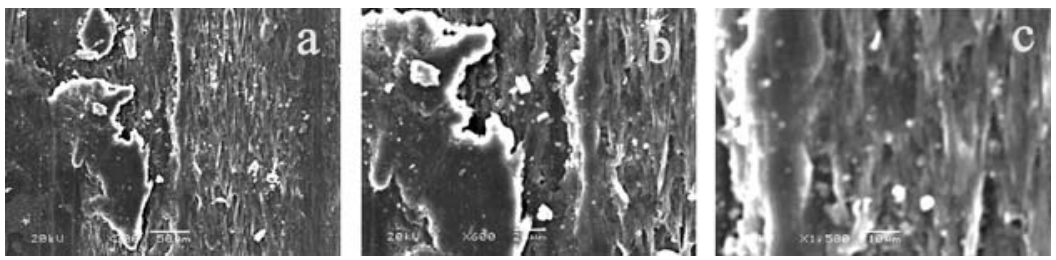

Figure 10. SEM morphologies of the worn surface of pure PI sliding under the load of $500 \mathrm{~N}$ and the velocity of $0.431 \mathrm{~m} / \mathrm{s}$; a) $300 \times$, b) $600 \times$, c) $1500 \times$

It can be seen from Figure 10 that the pure PI might experience melting and many peeling-off on the worn surface under a higher load. The friction may produce a great deal of heat and cause the temperature between the worn surface and the counterpart increased drastically under a higher load. At a higher temperature, the pure PI molecular relaxation changed and more chains were mobile and transited from the glassy state (with high strength, high stiffness) into the rubbery state (with lower strength and lower stiffness) and easier transferred to the counterpart surface, thus a smoother worn surface and high wear rate of pure PI were observed.

\section{Conclusions}

(1) The incorporation of the treated and untreated carbon fibers contributed to the different change of tribological behaviors of PI composites. In the tested system regarding the friction and wear the optimum content of carbon fibers was about $15 \mathrm{wt} \%$.

(2) The reinforcement of $\mathrm{HNO}_{3}$ treated carbon fibers was better than that of the untreated ones. After treated by $\mathrm{HNO}_{3}$, active groups or active sites for van der Waals and hydrogen bonding on the surfaces of carbon fibers increased, which can improve the interfacial adhesion between the fibers and the surrounding PI matrix and had a dominant effect on the friction and wear behaviors of polyimide composites.

(3) With increasing load, both the friction coefficient and the specific wear rates of the CFN-PI composites decreased. As for the pure PI, the friction coefficient decreased greatly, while the specific wear rate increased drastically owing to the micro-melting and mechanical deterioration caused by friction heat under a higher load.

\section{Acknowledgements}

The authors would like to acknowledge the financial support of the Innovative Group Foundation from NSFC (Grant No. 50421502) and the National Natural Science Foundation of China (Grant No.50475128).

\section{References}

[1] Donnet J. B., Bansal R. C.: Carbon Fibers, Dekker, New York (1990).

[2] Ogawa H., Satio.: Oxidation behavior of polyacrylonitrile fibers evaluated by new stabilization index. Carbon, 33, 783-788 (1995).

[3] Wu Z. H., Pittman C. U., Gardner S. D.: Nitric acid oxidation of carbon fibers and the effects of subsequent treatment in refluxing aqueous $\mathrm{NaOH}$. Carbon, 33, 597-605 (1995).

[4] Toyoda M., Katoh H., Inagaki M.: Intercalation of nitric acid into carbon fibers. Carbon, 39, 2231-2237 (2001).

[5] Basova Y. V., Hatori H., Yamada Y., Miyashita K.: Effect of oxidation-reduction surface treatment on the electrochemical behavior of PAN-based carbon fibers. Electrochemistry Communications, 1, 540-544 (1999).

[6] Theodoridou E., Jannakoudakis A. D., Jannakoudakis P. D., Andonoglou P., Besenhard J. O.: Electrochemical oxidation of PAN- and pitch-based carbon fibers and deposition of palladium on the obtained materials. Synthetic Metals, 87, 225-229 (1997).

[7] Yue Z. R., Jiang W., Wang L., Gardner S. D., Pittman C. U.: Surface characterization of electrochemically oxidized carbon fibers. Carbon, 37, 1785-1796 (1999).

[8] Pittman C. U., Jiang W., Yue Z. R., Gardner S., Wang L., Toghiani H., Leon C. A.: Surface properties of electrochemically oxidized carbon fibers. Carbon, 37, 1797-1805 (1999).

[9] Wu G. M.: Oxygen plasma treatment of high performance fibers for composites. Materials Chemistry and Physics, 85, 81-87 (2004).

[10] Bahadur S., Polineni V. K.: Tribological studies of glass fabric-reinforced polyamide composites filled with $\mathrm{CuO}$ and PTFE. Wear, 200, 95-104 (1996).

[11] Price D M.: Vapor Pressure Determination by Thermogravimetry. Thermochimica Acta, 367-368, 253 262, (2001). 
[12] Yudin V. E., Goykhman M. Y., Balik K., Glogar P., Gubanova G. N., Kudriavtsev V. V.: Carbonization behaviour of some polyimide resins reinforced with carbon fibers. Carbon, 38, 5-12 (2000).

[13] Lu Z. P., Friedrich K.: On sliding friction and wear of PEEK and its composites. Wear, 181-183, 624631(1995).

[14] Friedrich K., Karger-Kocsis K., Lu Z. P.: Effects of steel counterface roughness and temperature on the friction and wear of $\mathrm{PE}(\mathrm{E}) \mathrm{K}$ composites under dry sliding conditions. Wear, 148, 235-247 (1991).
[15] Li J. Q., Huang Y. D., Xu Z. W., Wang Z.: Highenergy radiation technique treat on the surface of carbon fiber. Materials Chemistry and Physics, 94, 315321 (2005).

[16] Wu S.: Polymer interface and adhesion. Marcel Dekker, New York (1982).

[17] Jang B. Z.: Control of interfacial adhesion in continuous carbon and kevlar fiber reinforced polymer composites. Composites Science and Technology, 44, 333-349 (1992). 